\title{
Mild Solutions for Nonlocal Impulsive Fractional Semilinear Differential Inclusions with Delay in Banach Spaces
}

\author{
Ahmed Gamal Ibrahim, Nawal Abdulwahab Al Sarori \\ Mathematics Department, Faculty of Science, King Faisal University, Al-Ahsa, KSA \\ Email: agamal2000@yahoo.com,n_alsarori@yahoo.com
}

Received March 19, 2013; revised April 19, 2013; accepted April 26, 2013

Copyright (C) 2013 Ahmed Gamal Ibrahim, Nawal Abdulwahab Al Sarori. This is an open access article distributed under the Creative Commons Attribution License, which permits unrestricted use, distribution, and reproduction in any medium, provided the original work is properly cited.

\begin{abstract}
In this paper, we give various existence results concerning the existence of mild solutions for nonlocal impulsive differential inclusions with delay and of fractional order in Caputo sense in Banach space. We consider the case when the values of the orient field are convex as well as nonconvex. Our obtained results improve and generalize many results proved in recent papers.
\end{abstract}

Keywords: Fractional Differential Inclusions; Impulsive Semilinear Functional Differential Inclusions; The Infinitesimal Generator of a Semigroup; Nonlocal Conditions; Mild Solutions

\section{Introduction}

During the past two decades, fractional differential equations and fractional differential inclusions have gained considerable importance due to their applications in various fields, such as physics, mechanics and engineering. For some of these applications, one can see [1-4] and the references therein. El Sayed et al. [5] initiated the study of fractional multi-valued differential inclusions. Recently, some basic theory for initial-value problems for fractional differential equations and inclusions was discussed by [6-14].

The theory of impulsive differential equations and impulsive differential inclusions has been an object interest because of its wide applications in physics, biology, engineering, medical fields, industry and technology. The reason for this applicability arises from the fact that impulsive differential problems are an appropriate model for describing process which at certain moments change their state rapidly and which cannot described using the classical differential problems. For some of these applications we refer to [15-17]. During the last ten years, impulsive differential inclusions with different conditions have intensely student by many mathematicians. At present, the foundations of the general theory of impulsive differential equations and inclusions are already laid, and many of them are investigated in details in the book of
Benchohra et al. [18].

Moreover, a strong motivation for investigating the nonlocal Cauchy problems, which is a generalization for the classical Cauchy problems with initial condition, comes from physical problems. For example, it used to determine the unknown physical parameters in some inverse heat condition problems. The nonlocal condition can be applied in physics with better effect than the classical initial condition $x(0)=x_{0}$. For example, $g(x)$ may be given by

$$
g(x)=\sum_{i=1}^{i=m} c_{i} x\left(t_{i}\right),
$$

where $c_{i}(i=1,2, \cdots, m)$ are given constants and $0<t_{1}$ $<t_{2}<\cdots<t_{n}<b$. For the applications of nonlocal conditions problems we refer to $[19,20]$. In the few past years, several papers have been devoted to study the existence of solutions for differential equations or differential inclusions with nonlocal conditions [21-23]. For impulsive differential equation or inclusions with nonlocal conditions of order one we refer to [22,23]. For impulsive differential equation or inclusions of fractional order we refer to [10,24-27] and the references therein.

In this paper we are concerned with the existence of mild solution to the following nonlocal impulsive semilinear differential inclusions with delay and of order $\alpha \in(0,1)$ of the type 


$$
\left\{\begin{array}{l}
{ }^{c} D^{\alpha} x(t) \in A x(t)+F(t, \tau(t) x), \text { a.e. on } J-\left\{t_{1}, t_{2}, \cdots, t_{m}\right\}, \\
x(t)=\psi(t)-g(x), t \in[-r, 0], \\
x\left(t_{i}^{+}\right)=x\left(t_{i}\right)+I_{i}\left(x\left(t_{i}\right)\right), i=1,2, \cdots, m,
\end{array}\right.
$$

where $J=[0, b], r, b>0,{ }^{c} D^{\alpha} x(t)$ is the Caputo derivative of order $\alpha, A: D(A) \subseteq E \rightarrow E$ is the infinitesimal generator of a $C_{0}$ - semigroup $\{T(t), t \geq 0\}$ on a real separable Banach space $E, \quad F: J \times \Phi \rightarrow 2^{E}$ be a multi-function,

$0=t_{0}<t_{1}<\cdots<t_{m}<t_{m+1}=b, \psi:[-r, 0] \rightarrow E$ is a given continuous function, $g: \mathcal{H} \rightarrow E$, is a nonlinear function related to the nonlocal condition at the origin,

$I_{i}: E \rightarrow E(i=1,2, \cdots, m)$ impulsive functions which characterize the jump of the solutions at impulse points, and $x\left(t_{i}^{+}\right),\left(t_{i}^{-}\right)$are the right and left limits of $x$ at the point $t_{i}$ respectively. Finally, for any $t \in J$, $\tau(t): \mathcal{H} \rightarrow \Phi$ defined by

$$
x(\theta)=x(t+\theta), \theta \in[-r, 0], x \in \mathcal{H},
$$

where $\Phi$ and $\mathcal{H}$ will define in the next section.

To study the theory of abstract impulsive differential inclusions with fractional order, the first step is how to define the mild solution. Mophou [24] firstly introduced a concept on a mild solution which was inspired by Jaradat et al. [25]. However, it does not incorporate the memory effects involved in fractional calculus and impulsive conditions. Wang et al. [10] introduced a new concept of $P C$-mild solutions for (1.1) without delay and derived existence and uniqueness results concerning the $P C$-mild solutions for (1.1) when $F$ is a Lipschitz single-valued function or continuous and maps bounded sets into bounded sets and $T(t), t>0$ is compact.

In order to do a comparison between our obtained results in this paper and the known recent results in the same domain, we refer to: Ouahab [9] proved a version of Fillippov's theorem for (1.1) without impulse, without delay and $A$ is an almost sectorial operator, Wang et al. [11] proved existence and controllability results for (1.1) without impulse, without delay and with local condition, Zhang et al. [12] considered the problem (1.1) without impulse, without delay, $F$ is a single-valued function and $\{T(t), t>0\}$ is strongly equicontinuous $C_{0}$-semigroup, Zhou et al. [13,14] introduced a suitable definition of mild solution for (1.1) based on Laplace transformation and probability density functions for (1.1) when $F$ is single-valued function and without impulse, Cardinali et al. [22] proved the existence of mild solutions to the problem (1.1) without delay, when $\alpha=1$ and the multivalued function $F$ satisfies the lower Scorza-Dragoni property and $\{A(t)\}_{t \geq 0}$ is a family of linear operator, generating a strongly continuous evolution operators, Fan [23] studied a nonlocal Cauchy problem in the presence of impulses, governed by autonomous semilinear differential equation, Dads et al. [26] and Henderson et al. [27] considered the problem (1.1) when $A=0$. Among the previous works, little is concerned with nonlocal fractional differential inclusions with impulses and with delay.

In Section 3 in this paper, motivated by the works mentioned above, we derive various existence results of mild solutions for (1.1) when the values of the orient field are convex as well as non-convex.

The paper is organized as follows: In Section 2, we collect some background material and lemmas to be used later. In Section 3, we prove three existence results for (1.1). We adopt the definition of mild solution introduced by Wang et al. [10]. Our basic tools are the properties of multi-functions, methods and results for semilinear differential inclusions, and fixed point techniques.

\section{Preliminaries and Notations}

Let $C(J, E)$ the space of $E$-valued continuous functions on $J$ with the uniform norm

$\|x\|=\sup \{\|x(t)\|, t \in J\}, L^{1}(J, E)$ the space of $E$-valued Bochner integrable functions on $J$ with the norm $\|f\|_{L^{1}(J, E)}=\int_{0}^{b}\|f(t)\| \mathrm{d} t, P_{b}(E)=\{B \subseteq E: B$ is nonempty and bounded $\}, P_{c l}(E)=\{B \subseteq E: B$ is nonempty and closed $\}, P_{k}(E)=\{B \subseteq E: B$ is nonempty and compact $\}, P_{c l, c v}(E)=\{B \subseteq E: B$ is nonempty, closed and convex $\}, P_{c k}(E)=\{B \subseteq E: B$ is nonempty, convex and compact $\}, \operatorname{conv}(B)$ (respectively,

$\overline{\operatorname{conv}}(B)$ ) be the convex hull (respectively, convex closed hull in $E$ ) of a subset $B$.

Definition 1 ([28]). A semigroup $T(t), 0 \leq t<\infty$, of bounded linear operators on a Banach space $X$ is said to be

1) uniformly continuous if

$$
\operatorname{Lim}_{t \downarrow 0}\|T(t)-I\|=0,
$$

where $I$ is the identity operator.

2) strongly continuous if

$$
\lim _{t \downarrow 0} T(t) x=x \text {, for every } x \in X .
$$

A strongly continuous semigroup of bounded linear operators on $X$ will be called a semigroup of class $C_{0}$ or simply a $C_{0}$-semigroup. It is known that if $T(t), 0 \leq t<\infty$ is a $C_{0}$-semigroup, then there exist constants $\omega \geq 0$ and $M \geq 1$ such that 


$$
\|T(t)\| \leq M \mathrm{e}^{\omega t} \text {, for } 0 \leq t<\infty .
$$

A $C_{0}$-semigroup $T(t), 0 \leq t<\infty$ is called compact if for every $t>0, T(t)$ is compact. It is known that ([28], Theorem 3.2) every compact $C_{0}$ - semigroup is uniformly continuous.

Definition 2 ([28]). Let $T(t), 0 \leq t<\infty$, be a semigroup of bounded linear operators on a Banach space $X$. The linear operator $A$ defined by

$$
D(A)=\left\{x \in X: \lim _{t \downarrow 0} \frac{T(t) x-x}{t} \text { exists }\right\}
$$

and

$$
A x=\lim _{t \downarrow 0} \frac{T(t) x-x}{t}
$$

is called the infinitesimal generator of the semigroup $T(t), D(A)$ is the domain of $A$.

Definition 3 ([29-33]). Let $X$ and $Y$ be two topological spaces. A multifunction $G: X \rightarrow P(Y)$ is said to be upper semicontinuous (u.s.c.) if

$G^{-1}(V)=\{x \in X: G(x) \subseteq V\}$ is an open subset of $X$ for every open $V \subseteq Y$. $G$ is said to be lower semicontinuous (l.s.c.) if $G^{+1}(V)=\{x \in X: G(x) \cap V \neq \phi\}$ is an open subset of $X$ for every open $V \subseteq Y$. $G$ is called closed if its graph

$\Gamma_{G}=\{(x, y) \in X \times Y: y \in G(x)\}$ is closed subset of the topological space $X \times Y$. G is said to be completely continuous if $G(B)$ is relatively compact for every bounded subset $B$ of $X$. If the multifunction $G$ is completely continuous with non empty compact values, then $G$ is u.s.c. if and only if $G$ is closed.

Lemma 1 ([29], Theorem 8.2.8). Let $(\Omega, A, \mu)$ be a complete $\sigma$-finite measure space, $X$ a complete separable metric space and $F: \Omega \rightarrow 2^{X}$ be a measurable multivalued function with non empty closed images. Consider a multivalued function $G$ from $: \Omega \times X$ to $P(Y), \quad Y$ is a complete separable metric space such that for every $x \in X$ the multivalued function $w \rightarrow G(w, x)$ is measurable and for every $w \in \Omega$ the multivalued function $x \rightarrow G(w, x)$ is continuous. Then the multivalued function $w \rightarrow \overline{G(w, F(w))}$ is measurable. In particular for every measurable singlevalued function $z: \Omega \rightarrow X$, the multivalued function $w \rightarrow G(w, z(w))$ is measurable and for every Caratheodory single-valued function $\varphi: \Omega \times X \rightarrow Y$, the multivalued function $w \rightarrow \overline{\varphi(w, F(w))}$ is measurable.

Definition 4 A nonempty subset $M \subseteq L^{1}(J, E)$ is said to be decomposable provided for every $f, g \in M$ and each Lebesgue measurable set $Z$ in $J$,

$f \chi_{Z}+g \chi_{(J-Z)} \in M$, where $\chi_{Z}$ is the characteristic function of the set $Z$.

Definition 5 A sequence $\left\{f_{n}: n \in \mathbb{N}\right\} \subset L^{1}(J, E)$ is said to be semi-compact if:

1) It is integrably bounded, i.e. there is $q \in L^{1}\left(J, \mathbb{R}^{+}\right)$ such that

$$
\left\|f_{n}(t)\right\| \leq q(t) \text { a.e. } t \in J .
$$

2)The set $\left\{f_{n}(t): n \in \mathbb{N}\right\}$ is relatively compact in $E$ a.e. $t \in J$.

We recall one fundamental result which follows from Dunford-Pettis Theorem.

Lemma 2 ([33]). Every semi-compact sequence in $L^{1}(J, E)$ is weakly compact in $L^{1}(J, E)$.

For more about multifunctions we refer to [29-33].

Lemma 3 ([11], lemma 2.10). For $\tau \in(0,1]$ and $0<e \leq c$, we have $\left|e^{\tau}-c^{\tau}\right| \leq(c-e)^{\tau}$.

Definition 6 According to the Riemann -Liouville approach, the fractional integral of order $\alpha \in(0,1)$ of a function $f \in L^{1}(J, E)$ is defined by

$$
I^{\alpha} f(t)=\int_{0}^{t} \frac{(t-s)^{\alpha-1}}{\Gamma(\alpha)} f(s) \mathrm{d} s, t>0,
$$

provided the right side is defined on $J$, where $\Gamma$ is the Euler gamma function defined by $\Gamma(\alpha)=\int_{0}^{\infty} t^{\alpha-1} \mathrm{e}^{-t} \mathrm{~d} t$.

Definition 7 The Caputo derivative of order $\alpha \in(0,1)$ of a continuously differentiable function $f: J \rightarrow E$ is defined by

$$
{ }^{c} D^{\alpha} f(t)=\frac{1}{\Gamma(1-\alpha)} \int_{0}^{t}(t-s)^{-\alpha} f^{(1)}(s) \mathrm{d} s=I^{(1-\alpha)} f^{(1)}(t) .
$$

Note that the integrals appear in the two previous definitions are taken in Bochner' sense and ${ }^{c} D^{\alpha} I^{\alpha} f(t)$ $=f(t)$ for all $t \in J$. For more informations about the fractional calculus we refer to [2,4].

Definition 8 ([14], Lemma 3.1 and Definition 3.1, see also [11-13]). Let $h: J \rightarrow E$. A function $x \in C(J, E)$ is said to be a mild solution of the following system:

$$
\left\{\begin{array}{l}
{ }^{c} D^{\alpha} x(t)=A(t) x(t)+h(t), t \in J, \\
x(0)=x_{0} \in E,
\end{array}\right.
$$

if it satisfies the following integral equation

$$
\begin{aligned}
& x(t)=K_{1}(t)\left(x_{0}\right)+\int_{0}^{t}(t-s)^{\alpha-1} K_{2}(t-s) h(s) \mathrm{d} s, \\
& t \in J,
\end{aligned}
$$

where

$$
\begin{aligned}
K_{1}(t) & =\int_{0}^{\infty} \xi_{\alpha}(\theta) T\left(t^{\alpha} \theta\right) \mathrm{d} \theta, K_{2}(t) \\
& =\alpha \int_{0}^{\infty} \theta \xi_{\alpha}(\theta) T\left(t^{\alpha} \theta\right) \mathrm{d} \theta, \xi_{\alpha}(\theta) \\
& =\frac{1}{\alpha} \theta^{-1-\frac{1}{\alpha}} w_{\alpha}\left(\theta^{-\frac{1}{\alpha}}\right) \geq 0,
\end{aligned}
$$


$w_{\alpha}(\theta)=\frac{1}{\pi} \sum_{n=1}^{\infty}(-1)^{n-1} \theta^{-\alpha n-1} \frac{\Gamma(n \alpha+1)}{n !} \sin (n \pi \alpha)$,

$\theta \in(0, \infty)$ and $\xi_{\alpha}$ is a probability density function defined on $(0, \infty)$, that is $\int_{0}^{\infty} \xi_{\alpha}(\theta) \mathrm{d} \theta=1$. Note that the function must be chosen such that the integral appears in (2.2) is well be defined.

Remark 1 Since $K_{1}(),. K_{2}($.$) are associated with$ the numbrer $\alpha$, there are no analogue of the semigroup property, i.e. $K_{1}(t+s) \neq K_{1}(t) K_{1}(s)$, $K_{2}(t+s) \neq K_{2}(t) K_{2}(s)$.

In the following we recall the properties of $K_{1}($.$) ,$ $K_{2}($.$) .$

Lemma 4 ([14], Lemma 3.2, Lemma 3.3 and Lemma 3.5)
1) For any fixed $t \geq 0, K_{1}(t), K_{2}(t)$ are linear bounded operators.

2) For $\gamma \in[0,1], \int_{0}^{\infty} \theta^{\gamma} \xi_{\alpha}(\theta) \mathrm{d} \theta=\frac{\Gamma(1+\gamma)}{\Gamma(1+\alpha \gamma)}$.

3) If $\|T(t)\| \leq M, t \geq 0$, then for any $x \in E,\left\|K_{1}(t) x\right\| \leq M\|x\|$ and $\left\|K_{2}(t) x\right\| \leq \frac{M}{\Gamma(\alpha)}\|x\|$.

4) For any fixed $t \geq 0, K_{1}(t), K_{2}(t)$ are strongly continuous.

5) If $T(t), t>0$ is compact, then $K_{1}(t)$ and $K_{2}(t)$ are compact.

In order to define the concept of mild solution of (1.1), let $J_{0}=\left[0, t_{1}\right], J_{i}=\left(t_{i}, t_{i+1}\right], \quad i=1,2, \cdots, m$ and consider the set of functions:

$\Phi=\{\psi:[-r, 0] \rightarrow E: \psi$ is continuous everywhere except for a finite number of points $s$ at which $\psi(s-)$ and $\psi(s+)$ exist and $\psi(s)=\psi(s-)\}$,

$P C(J, E)=\left\{x: J \rightarrow E: x_{\mid J_{i}} \in C\left(J_{i}, E\right), i=0,1,2, \cdots, m\right.$ and $x\left(t_{i}^{+}\right)$and $x\left(t_{i}^{-}\right)$exist for each $\left.i=1,2, \cdots, m\right\}$,

and

$$
\mathcal{H}=\left\{x:[-r, b] \rightarrow E:\left.x\right|_{[-r, 0]} \in \Phi, x_{\mid J_{i}} \in C\left(J_{i}, E\right), i=0,1,2, \cdots, m \text { and } x\left(t_{i}^{+}\right) \text {and } x\left(t_{i}^{-}\right) \text {exist for each } i=1,2, \cdots, m\right\} .
$$

It is easy to check that $\Phi$ are $\mathcal{H}$ are Banach spaces endowed with the norms

$$
\|\psi\|_{\Phi}=\max \{\|x(t)\|: t \in[-r, 0]\},
$$

and

$$
\begin{aligned}
&\|x\|_{\mathcal{H}}=\max \{\|x(t)\|: t \in[-r, b]\} . \begin{array}{c}
t-r \text { up the present time } t \text {. For } \\
\text { and for any } i=0,1,2, \cdots, m, \text { let }
\end{array} \\
& B_{\mid \bar{J}_{i}}=\left\{x^{*}: \bar{J}_{i} \rightarrow E: x^{*}(t)=x(t), t \in J_{i} \text { and } x^{*}\left(t_{i}\right)=x\left(t_{i}^{+}\right), x \in B\right\}, i=0,1, \cdots, m .
\end{aligned}
$$

Here $\tau(t) x$ represents the history of the state time $t-r$ up the present time $t$. For any subset $B \subseteq \mathcal{H}$

Let us recall the concept of mild solutions, introduced Of course $B_{\mid \overline{J_{0}}}=\left\{x_{\mid \overline{J_{0}}}: x \in B\right\}$.

$$
\left\{\begin{array}{l}
{ }^{c} D^{\alpha} x(t)=A x(t)+f(t, x(t)), \text { a.e. on } J-\left\{t_{1}, t_{2}, \cdots, t_{m}\right\} \\
x(0)=x_{0} \\
x\left(t_{i}^{+}\right)=x\left(t_{i}\right)+y_{i}, i=1,2, \cdots, m,
\end{array}\right.
$$

where $f: I \times E \rightarrow E, y_{i} \in E, i=1,2, \cdots, m$.

At first Wang et al. [10] considered the following nonhomogeneous impulsive fractional equation

$$
\left\{\begin{array}{l}
{ }^{c} D^{\alpha} x(t)=A x(t)+h(t), t \in J-\left\{t_{1}, t_{2}, \cdots, t_{m}\right\}, \\
x(0)=x_{0}, \\
x\left(t_{i}^{+}\right)=x\left(t_{i}\right)+y_{i}, i=1,2, \cdots, m,
\end{array}\right.
$$

where $h \in P C(J, E)$ and $y_{i} \in E$. It is easily observe that $x$ can be decomposed to $v+w$ where $v \in C(J, E)$ is the continuous mild solution for

$$
\left\{\begin{array}{l}
{ }^{c} D^{\alpha} v(t)=A v(t)+h(t), t \in J \\
v(0)=x_{0}
\end{array}\right.
$$

and $w \in P C(J, E)$ is the mild solution for the impul- 
sive evolution equation

$$
\left\{\begin{array}{l}
{ }^{c} D^{\alpha} w(t)=A w(t), t \in J-\left\{t_{1}, t_{2}, \cdots, t_{m}\right\}, \\
w(0)=0, \\
w\left(t_{i}^{+}\right)=w\left(t_{i}\right)+y_{i}, i=1,2, \cdots, m .
\end{array}\right.
$$

Indeed, by adding together (2.5) with (2.6), it follows (2.4). Note $v$ is continuous, so $v\left(t_{i}^{+}\right)=v\left(t_{i}\right)$, $i=1,2, \cdots, m$. On the other hand, any solution of (2.4) can be decomposed to (2.5) and (2.6). By Definition 9, a mild solution of (2.5) is given by

$$
\begin{aligned}
v(t) & =K_{1}(t)\left(x_{0}\right) \\
& +\int_{0}^{t}(t-s)^{\alpha-1} K_{2}(t-s) h(s) \mathrm{d} s, t \in J .
\end{aligned}
$$

Now we rewrite system (2.6) in the equivalent integral equation

$$
w(t)=\left\{\begin{array}{l}
\frac{1}{\Gamma(\alpha)} \int_{0}^{t}(t-s)^{\alpha-1} A w(s) \mathrm{d} s, t \in J_{0}, \\
y_{1}+\frac{1}{\Gamma(\alpha)} \int_{0}^{t}(t-s)^{\alpha-1} A w(s) \mathrm{d} s, t \in J_{1}, \\
y_{1}+y_{2}+\frac{1}{\Gamma(\alpha)} \int_{0}^{t}(t-s)^{\alpha-1} A w(s) \mathrm{d} s, t \in J_{2}, \\
\vdots \\
\sum_{i=1}^{i=m} y_{i}+\frac{1}{\Gamma(\alpha)} \int_{0}^{t}(t-s)^{\alpha-1} A w(s) \mathrm{d} s, t \in J_{m} .
\end{array}\right.
$$

The above equation can be expressed as

$$
\begin{aligned}
w(t) & =\sum_{i=1}^{i=m} y_{i} \chi_{i}(t) \\
& +\frac{1}{\Gamma(\alpha)} \int_{0}^{t}(t-s)^{\alpha-1} A w(s) \mathrm{d} s, t \in J,
\end{aligned}
$$

where

$$
\chi_{i}(t)= \begin{cases}0, & \text { for } t \in\left[0, t_{i}\right) \\ 1, & \text { for } t \in\left[t_{i}, b\right] .\end{cases}
$$

We apply the Laplace transform for (2.8) to get (see, [25])

$$
\begin{gathered}
\left.u(\lambda)=\sum_{i=1}^{i=m} y_{i} \frac{\mathrm{e}^{-t_{i} \lambda}}{\lambda}+\frac{1}{\lambda^{\alpha}} A(u(\lambda)), \quad \text { by } \quad \text { By } 2.7\right) \text { and (2.10), the mild solutic } \\
x(t)=K_{1}(t)\left(x_{0}\right)+\sum_{i=1}^{i=m} \chi_{i}(t) K_{1}\left(t-t_{i}\right) y_{i}+\int_{0}^{t}(t-s)^{\alpha-1} K_{2}(t-s) h(s) \mathrm{d} s, t \in J .
\end{gathered}
$$

By (2.7) and (2.10), the mild solution of (2.4) is given

of the system (2.3) we mean a function $x \in P C(J, E)$ which satisfies the following integral equation

$$
u(\lambda)=\sum_{i=1}^{i=m} \mathrm{e}^{-t_{i} \lambda} \lambda^{\alpha-1}\left(\lambda^{\alpha} I-A\right)^{-1} y_{i} .
$$

Note that the Laplace transform for $K_{1}(t) y_{i}$ is $\lambda^{\alpha-1}\left(\lambda^{\alpha} I-A\right)^{-1} y_{i}$. Thus we can derive the mild solution of (2.6) as

$$
w(t)=\sum_{i=1}^{i=m} \chi_{i}(t) K_{1}\left(t-t_{i}\right) y_{i} .
$$

By using the above results, we can write the following definition of mild solution of the system (2.3).

Definition 9 ([10], Definition 3.1). By a mild solution

$$
x(t)=\left\{\begin{array}{l}
K_{1}(t)\left(x_{0}\right)+\int_{0}^{t}(t-s)^{\alpha-1} K_{2}(t-s) f(s, x(s)) \mathrm{d} s, t \in J_{0}, \\
K_{1}(t)\left(x_{0}\right)+K_{1}\left(t-t_{1}\right) y_{1}+\int_{0}^{t}(t-s)^{\alpha-1} K_{2}(t-s) f(s, x(s)) \mathrm{d} s, t \in J_{1}, \\
\vdots \\
K_{1}(t)\left(x_{0}\right)+\sum_{i=1}^{i=m} K_{1}\left(t-t_{i}\right) y_{i}+\int_{0}^{t}(t-s)^{\alpha-1} K_{2}(t-s) f(s, x(s)) \mathrm{d} s, t \in J_{m} .
\end{array}\right.
$$


Now we can give the concept of mild solution for our considered problem (1.1).

Definition 10 By a mild solution for (1.1), we mean a function $x \in \mathcal{H}$ which satisfies the following integral equation

$$
x(t)=\left\{\begin{array}{l}
\psi(t)-g(x), t \in[-r, 0] \\
K_{1}(t)(\psi(0)-g(x))+\int_{0}^{t}(t-s)^{\alpha-1} K_{2}(t-s) f(s) \mathrm{d} s, t \in J_{0}, \\
K_{1}(t)(\psi(0)-g(x))+K_{1}\left(t-t_{1}\right) y_{1}+\int_{0}^{t}(t-s)^{\alpha-1} K_{2}(t-s) f(s) \mathrm{d} s, t \in J_{1}, \\
\quad \vdots \\
K_{1}(t)(\psi(0)-g(x))+\sum_{i=1}^{i=m} K_{1}\left(t-t_{i}\right) y_{i}+\int_{0}^{t}(t-s)^{\alpha-1} K_{2}(t-s) f(s) \mathrm{d} s, t \in J_{m},
\end{array}\right.
$$

where $y_{i}=I_{i}\left(x\left(t_{i}^{-}\right)\right), i=1, \cdots, m$ and $f$ is an integrable selection for $F(., \tau() x$.$) .$

Remark 2 It is easily to see that the solution given by (2.11) satisfies the relation

$$
x\left(t_{i}^{+}\right)=x\left(t_{i}\right)+I_{i}\left(x\left(t_{i}\right)\right), i=1,2, \cdots, m .
$$

Remark 3 If $I_{i}\left(x\left(t_{i}^{-}\right)\right)=0$, for all $i=1, \cdots, m, g=0$ and if there is no delay then Formula (2.11) will take the form

$$
x(t)=K_{1}(t) x_{0}+\int_{0}^{t}(t-s)^{\alpha-1} K_{2}(t-s) f(s) \mathrm{d} s, t \in J .
$$

This means that when there is no neither impulse nor delay in the problem (1.1), its solution is equal to the formula given in (2.2).

Theorem 1 ([34]). Let $W$ be a nonempty subset of a Banach space $E$, which is bounded, closed and convex. Suppose $R: W \rightarrow 2^{E}$ is u.s.c. with closed, convex values, and such that $R(W) \subseteq W$ and $R(W)$ is compact. Then $R$ has a fixed point

The following fixed point theorem for contraction multivalued is proved by Govitz and Nadler [35].

Theorem 2 Let $(X, d)$ be a complete metric space. If $R: X \rightarrow P_{c l}(X)$ is contraction, then $R$ has a fixed point.

Theorem 3 ([36], Corollary 3.3.1) (Schauder fixed point theorem). Let $E$ be a Banach space, $B$ a nonempty, convex, closed and bounded subset of $E$ and $f: B \rightarrow B$ be continuous. If $f$ is compact or $B$ is compact, then $f$ has a fixed point.

\section{Existence Results for the Problem (1.1)}

In this section, we give the main results of mild solutions of (1.1).

\subsection{Convex Case}

In the following Theorem we derive the first existence result concerning the mild solution for the problem (1.1).
Theorem 4 Let $F: J \times \Phi \rightarrow P_{c k}(E)$ be a multifunction. Assume the following conditions:

$\left(\mathrm{H}_{1}\right) \mathrm{A}$ is the infinitesimal generator of a $C_{0}$-semigroup $\{T(t): t \geq 0\}$ and $T(t), t>0$ is compact.

$\left(\mathrm{H}_{2}\right)$ For every $h \in \Phi, t \rightarrow F(t, h)$ is measurable, for almost $t \in J, h \rightarrow F(t, h)$ is upper semi-continuous and for each $x \in \mathcal{H}$, the set

$S_{F(\cdot, \tau(\cdot) x)}^{1}=\left\{f \in L^{1}(J, E): f(t) \in F(t, \tau(t) x)\right.$, a.e. $\}$ is nonempty.

$\left(\mathrm{H}_{3}\right)$ There exist a function $\varphi \in L^{\frac{1}{q}}\left(J, \mathbb{R}^{+}\right)$, $0<q<\alpha$ such that for any $x \in \Phi$

$$
\sup \{\|z\|: z \in F(t, x)\} \leq \varphi(t)(1+x(0)) \text {, a.e. } t \in J \text {. }
$$

$\left(\mathrm{H}_{4}\right) \quad g: \mathcal{H} \rightarrow E$ is continuous, compact and there exist two positive numbers $a, d$ such that

$$
\|g(h)\| \leq a\|h\|+d, \forall h \in \mathcal{H} .
$$

$\left(\mathrm{H}_{5}\right)$ For every $i=1,2, \cdots, m, \quad I_{i}$ is continuous and compact and there exists a positive constant $h_{i}$ such that

$$
\left\|I_{i}(x)\right\| \leq h_{i}\|x\|, x \in E .
$$

Then, for a given continuous function $\psi:[-r, 0] \rightarrow E$, the problem (1.1) has a mild solution provided that there is $r>0$ such that

$$
\begin{aligned}
& (M+1)[\|\psi\|+a(r+\|\psi\|)+d] \\
& +M[h(r+\|\psi\|)+\eta(1+\|\psi\|+r)] \leq r .
\end{aligned}
$$

where, $M>0$ such that $\sup _{t \in J}\|T(t)\| \leq M$,

$$
h=\sum_{i=1}^{i=m} h_{i}, \eta=\frac{b^{\alpha-q}}{\Gamma(\alpha)(\varpi+1)^{1-q}}\|\varphi\|_{L^{\frac{1}{q}}\left(J, \mathbb{R}^{+}\right)}
$$

and $\varpi=\frac{\alpha-1}{1-q}$.

Proof. In view of $\left(\mathrm{H}_{2}\right)$, for each $x \in \mathcal{H}$, the set 


$$
\begin{gathered}
S_{F(\cdot, \tau(\cdot) x)}^{1}=\left\{f \in L^{1}(J, E): f(t) \in F(t, \tau(t) x), \text { a.e. } \quad \begin{array}{r}
\text { is nonempty. So, we can define a multifunction } \\
G: \mathcal{H} \rightarrow 2^{\mathcal{H}}, \text { as follows: } y \in G(x) \text { if and only if }
\end{array}\right. \\
y(t)=\left\{\begin{array}{l}
\psi(t)-g(x), t \in[-r, 0], \\
K_{1}(t)(\psi(0)-g(x))+\int_{0}^{t}(t-s)^{\alpha-1} K_{2}(t-s) f(s) \mathrm{d} s, t \in J_{0}, \\
K_{1}(t)(\psi(0)-g(x))+\sum_{k=1}^{k=i} K_{1}\left(t-t_{k}\right) I_{k}\left(x\left(t_{k}^{-}\right)\right) \\
+\int_{0}^{t}(t-s)^{\alpha-1} K_{2}(t-s) f(s) \mathrm{d} s, t \in J_{i}, i=1,2, \cdots, m .
\end{array}\right.
\end{gathered}
$$

where $f \in S_{F(\cdot, \tau(\cdot) x)}^{1}$. Obviously, every fixed point for $G$ is a mild solution for the problem (1.1). So, our goal is to apply Theorem 1 . The proof will be given in several steps.

Step 1. The values of $G$ are convex and closed subset in $\mathcal{H}$.

Since the values of $F$ are convex, it is easily to see

$$
y_{n}(t)=\left\{\begin{array}{l}
\psi(t)-g(x), t \in[-r, 0], \\
K_{1}(t)(\psi(0)-g(x))+\int_{0}^{t}(t-s)^{\alpha-1} K_{2}(t-s) f_{n}(s) \mathrm{d} s, t \in J_{0}, \\
K_{1}(t)(\psi(0)-g(x))+\sum_{k=1}^{k=i} K_{1}\left(t-t_{k}\right) I_{k}\left(x\left(t_{k}^{-}\right)\right) \\
\quad+\int_{0}^{t}(t-s)^{\alpha-1} K_{2}(t-s) f_{n}(s) \mathrm{d} s, t \in J_{i}, i=1,2, \cdots, m . .
\end{array}\right.
$$

Not that, from (3.1), for any $n \geq 1$, for almost $s \in J$

$$
\left\|f_{n}(s)\right\| \leq \varphi(s)(1+\|\tau(s) x(0)\|)=\varphi(s)(1+\|x(s)\|) \leq \varphi(s)\left(1+\|x\|_{\mathcal{H}}\right)
$$

This show that the set $\left\{f_{n}: n \geq 1\right\}$. is integrably bounded. Moreover, because

$\left\{f_{n}(t): n \geq 1\right\} \subset F(t, \tau(t) x)$, for a.e. $t \in J$, the set

$\left\{f_{n}(t): n \geq 1\right\}$ is relativity compact in $E$ for a.e. $t \in J$. Therefore, the set $\left\{f_{n}: n \geq 1\right\}$ is semi-compact and then, by Lemma 2 it is weakly compact in $L^{1}\left(J, \mathbb{R}^{+}\right)$. So, without loss of generality we can assume that $f_{n}$ converges weakly to a function $f \in L^{1}\left(J, \mathbb{R}^{+}\right)$. From Mazur's lemma, there is a sequence $\left(g_{n}\right), n \geq 1$ such that $\left\{g_{n}(t): n \geq 1\right\} \subseteq \overline{\operatorname{Conv}}\left\{f_{n}(t): n \geq 1\right\} ; t \in J$ and $g_{n}$ converges strongly to $f$. Since, the values of $F$ are convex, $g_{n} \in S_{F(., \tau(t) x)}^{1}$ and hence, by the compactness of $F(t, \tau(t) x), \quad f \in S_{F(., \tau(t) x)}^{1}$. Moreover, for every $t, s \in J, s \in(0, t]$ and for every $n \geq 1$,

$$
\begin{aligned}
& \left\|(t-s)^{\alpha-1} K_{2}(t-s) f_{n}(s)\right\| \\
& \leq|t-s|^{\alpha-1} \frac{M}{\Gamma(\alpha)} \varphi(s)\left(1+\|x\|_{\mathcal{H}}\right) \in L^{1}\left((0, t], R^{+}\right) .
\end{aligned}
$$

Therefore, by passing to the limit as $n \rightarrow \infty$ in (3.5), we obtain from the Lebesgue dominated convergence theorem that, for every $i=0,1, \cdots, m$,

$$
y(t)=\left\{\begin{array}{l}
\psi(t)-g(x), t \in[-r, 0] \\
K_{1}(t)(\psi(0)-g(x))+\int_{0}^{t}(t-s)^{\alpha-1} K_{2}(t-s) f(s) \mathrm{d} s, t \in J_{0}, \\
K_{1}(t)(\psi(0)-g(x))+\sum_{k=1}^{k=i} K_{1}\left(t-t_{k}\right) I_{k}\left(x\left(t_{k}^{-}\right)\right) \\
\quad+\int_{0}^{t}(t-s)^{\alpha-1} K_{2}(t-s) f(s) \mathrm{d} s, t \in J_{i}, i=1,2, \cdots, m .
\end{array}\right.
$$


Then $y \in G(x)$.

Step 2. We claim that $G\left(B_{r}\left(x^{*}\right)\right) \subseteq B_{r}\left(x^{*}\right)$, where

$$
x^{*}(t)= \begin{cases}\psi(t), & t \in[-r, 0] \\ \psi(0), & t \in J .\end{cases}
$$

and $B_{r}\left(x^{*}\right)=\left\{x \in \mathcal{H}:\left\|x-x^{*}\right\| \leq r\right\}$. To prove that, let $x \in B_{r}\left(x^{*}\right), y \in G(x)$ and $t \in J$. If $t \in[-r, 0]$, then by (3.2)

$$
\begin{aligned}
& \left\|y(t)-x^{*}(t)\right\| \leq\|g(x)\| \\
& \leq a\|x\|+d \leq a(\|\psi\|+r)+d .
\end{aligned}
$$

For $t \in J_{0}$. By using Lemma 4(3), (3.1), (3.2) and (3.5) we get

$$
\begin{aligned}
\left\|y(t)-x^{*}(t)\right\| & \leq\|\psi(0)\|+M(\|\psi(0)\|+a(r+\|\psi\|)+d)+\frac{M}{\Gamma(\alpha)}\left(1+\|x\|_{\mathcal{H}}\right) \int_{0}^{t}(t-s)^{\alpha-1} \varphi(s) \mathrm{d} s \\
& \leq\|\psi(0)\|+M(\|\psi\|+a(r+\|\psi\|)+d)+\frac{M}{\Gamma(\alpha)}(1+\|\psi\|+r)\|\varphi\|_{L^{\frac{1}{q}}}\left(J, \mathbb{R}^{+}\right)\left(\int_{0}^{t}(t-s)^{\frac{\alpha-1}{1-q}} \mathrm{~d} s\right)^{1-q} \\
& \leq\|\psi\|+M(\|\psi\|+a(r+\|\psi\|)+d)+\frac{b^{\alpha-q} M(1+\|\psi\|+r)}{\Gamma(\alpha)(\varpi+1)^{1-q}}\|\varphi\|_{L^{\frac{1}{q}}\left(J, \mathbb{R}^{+}\right)} \\
& \leq\|\psi\|+M(\|\psi\|+a(r+\|\psi\|)+d+(1+\|\psi\|+r) \eta) .
\end{aligned}
$$

Similarly, by using Lemma 4(3), (3.1), (3.2),(3.3) and (3.5) we have for $t \in J_{i}, \quad i=1,2, \cdots, m$,

$$
\|y(t)\| \leq\|\psi\|+M[\|\psi\|+a(r+\|\psi\|)+d+h(r+\|\psi\|)+\eta(1+\|\psi\|+r)] .
$$

Therefore, from (3.4).(3.6).(3.7) and (3.8), we conclude that $G\left(B_{r}\left(x^{*}\right)\right) \subseteq B_{r}\left(x^{*}\right)$.
Step 3. Let $B=G\left(B_{r}\left(x^{*}\right)\right)$. We claim that $B$ is equicontinuous, let $x \in B$ and $y \in G(x)$. According to the definition of $G$ we have

$$
y(t)=\left\{\begin{array}{l}
\psi(t)-g(x), t \in[-r, 0], \\
K_{1}(t)(\psi(0)-g(x))+\int_{0}^{t}(t-s)^{\alpha-1} K_{2}(t-s) f(s) \mathrm{d} s, t \in J_{0}, \\
K_{1}(t)(\psi(0)-g(x))+\sum_{k=1}^{k=i} K_{1}\left(t-t_{k}\right) I_{k}\left(x\left(t_{k}^{-}\right)\right)+\int_{0}^{t}(t-s)^{\alpha-1} K_{2}(t-s) f(s) \mathrm{d} s, t \in J_{i}, i \geq 1,
\end{array}\right.
$$

where $f \in S_{F(\cdot, \tau(\cdot) x)}^{1}$. By the continuity of $\psi$, we can see easily that if $t, t+\lambda \in[-r, 0]$, then

$$
\lim _{\lambda \rightarrow 0}\left\|y^{*}(t+\lambda)-y^{*}(t)\right\|=0 .
$$

To show that $B_{J}$, it suffices to verify that $B_{\overline{J_{i}}}$ is equicontinuous for every $i=0,1, \cdots, m$, where

$$
\left.\left.B=\left\{x^{*} \in C\left(\overline{J_{i}}, E\right): x^{*}(t)=x(t), t \in J_{i}=\right] t_{i}, t_{i+1}\right], x^{*}\left(t_{i}\right)=x\left(t_{i}^{+}\right), x \in B\right\} .
$$

We consider the following cases:

Case 1. Let $t=0, \lambda \in\left(0, t_{1}\right]$. In view of Holder's inequality we get

$$
\begin{aligned}
\left\|y^{*}(t+\lambda)-y^{*}(t)\right\| & =\|y(t+\lambda)-y(t)\|=\|y(\lambda)-y(0)\| \\
& \leq\left\|k_{1}(\lambda)(\psi(0)-g(x))-k_{1}(0)(\psi(0)-g(x))\right\|+\left\|\int_{0}^{\lambda}(\lambda-s)^{\alpha-1} k_{2}(\lambda-s) f(s) \mathrm{d} s\right\| \\
& \leq\left\|k_{1}(\lambda)-k_{1}(0)\right\|\|\psi(0)-g(x)\|+\frac{M(1+\|\psi\|+r)}{\Gamma(\alpha)}\|\varphi\|_{L^{\frac{1}{q}}\left(J, \mathbb{R}^{+}\right)}\left(\int_{0}^{\lambda}(\lambda-s)^{\frac{\alpha-1}{1-q}} \mathrm{~d} s\right)^{1-q} \\
& \leq\left\|k_{1}(\lambda)-k_{1}(0)\right\|\|\psi(0)-g(x)\|+\frac{M(1+\|\psi\|+r)}{\Gamma(\alpha)}\|\varphi\|_{L^{q}} \frac{1}{\left(J, \mathbb{R}^{+}\right)} \frac{\lambda^{\alpha-q}}{(\bar{\omega}+1)^{1-q}} .
\end{aligned}
$$


Since $T(\lambda)$ is compact, $K_{1}(\lambda)$ is also, (see, Lemma 4(v)), and hence, $K_{1}(h)$ is uniformly continuous on $J$ (see [28]). Therefore, the last inequality tends to zero as $\lambda \rightarrow 0$, independently of $x$.

Case 2. Let $t, t+\lambda$ be two points in $\left(0, t_{1}\right]$, then

$$
\begin{aligned}
& \left\|y^{*}(t+\lambda)-y^{*}(t)\right\|=\|y(t+\lambda)-y(t)\| \\
& \leq\left\|k_{1}(t+\lambda)(\psi(0)-g(x))-k_{1}(t)(\psi(0)-g(x))\right\| \\
& \quad+\left\|\int_{0}^{t+\lambda}(t+\lambda-s)^{\alpha-1} k_{2}(t+\lambda-s) f(s) \mathrm{d} s-\int_{0}^{t}(t-s)^{\alpha-1} k_{2}(t-s) f(s) \mathrm{d} s\right\| \\
& \leq G_{1}+G_{2}+G_{3}+G_{4},
\end{aligned}
$$

where

$$
\begin{aligned}
& G_{1}=\left\|k_{1}(t+\lambda)(\psi(0)-g(x))-k_{1}(t)(\psi(0)-g(x))\right\|, \\
& G_{2}=\left\|\int_{t}^{t+\lambda}(t+\lambda-s)^{\alpha-1} k_{2}(t+\lambda-s) f(s) \mathrm{d} s\right\|, \\
& G_{3}=\left\|\int_{0}^{t}\left[(t+\lambda-s)^{\alpha-1}-(t-s)^{\alpha-1}\right] k_{2}(t+\lambda-s) f(s) \mathrm{d} s\right\|,
\end{aligned}
$$$$
G_{4}=\left\|\int_{0}^{t}(t-s)^{\alpha-1}\left[k_{2}(t+\lambda-s)-k_{2}(t-s)\right] f(s) \mathrm{d} s\right\| .
$$

We only need to check $G_{i} \rightarrow 0$ as $\lambda \rightarrow 0$ for every $i=1,2,3,4$. At first, we note that, as we mention above the operators $K_{1}(t), t>0$ are uniformly continuous on and $J$. So, $\lim _{h \rightarrow 0} G_{1}=0$, independently of $x \in B_{r}\left(x^{*}\right)$.

For $G_{2}$, by the Holder inequality we have

$$
\begin{aligned}
\lim _{\lambda \rightarrow 0} G_{2} & =\lim _{\lambda \rightarrow 0}\left\|\int_{t}^{t+\lambda}(t+\lambda-s)^{\alpha-1} k_{2}(t+\lambda-s) f(s) \mathrm{d} s\right\| \\
& \leq \frac{M}{\Gamma(\alpha)} \lim _{\lambda \rightarrow 0} \int_{t}^{t+\lambda}(t+\lambda-s)^{\alpha-1}\|f(s)\| \mathrm{d} s \leq \frac{M(1+\|\psi\|+r)}{\Gamma(\alpha)} \lim _{\lambda \rightarrow 0} \int_{t}^{t+\lambda}(t+\lambda-s)^{\alpha-1} \varphi(s) \mathrm{d} s \\
& \left.\leq \frac{M(1+\|\psi\|+r)}{\Gamma(\alpha)} \lim _{\lambda \rightarrow 0}\left[\int_{t}^{t+\lambda}(t+\lambda-s)^{\frac{\alpha-1}{1-q}} \mathrm{~d} s\right]^{1-q}\|\varphi\|_{L^{q}} \frac{1}{\left(J, \mathbb{R}^{+}\right.}\right) \leq \frac{M(1+\|\psi\|+r)}{\Gamma(\alpha)} \lim _{\lambda \rightarrow 0}\left[\frac{\lambda^{(\bar{\omega}+1)}}{\bar{\omega}+1}\right]^{1-q}\|\varphi\|_{L^{\bar{q}}\left(J, \mathbb{R}^{+}\right)}=0,
\end{aligned}
$$

independently of $x \in B_{r}\left(x^{*}\right)$.

For $G_{3}$, we note that $\bar{\omega}=\frac{\alpha-1}{1-q} \in(-1,0)$, then for $s<t \leq t+\lambda$, we have $(t-s)^{\bar{\omega}} \geq(t+\lambda-s)^{\bar{\omega}}$. By applying Lemma 3 and taking into account $1-q \in(0,1)$ we get

$$
\begin{aligned}
& \left|\left[(t-s)^{\bar{\omega}}\right]^{1-q}-\left[(t+\lambda-s)^{\bar{\omega}}\right]^{1-q}\right| \\
& \leq\left[(t-s)^{\bar{\omega}}-(t+\lambda-s)^{\bar{\omega}}\right]^{1-q} .
\end{aligned}
$$

$$
\begin{aligned}
\lim _{\lambda \rightarrow 0} G_{3} & \leq \frac{M(1+\|\psi\|+r)}{\Gamma(\alpha)} \lim _{\lambda \rightarrow 0}\left[\int_{0}^{t}\left|(t-s)^{\alpha-1}-(t+\lambda-s)^{\alpha-1}\right|^{\frac{1}{1-q}} \mathrm{~d} s\right]^{1-q}\|\varphi\|_{L^{\frac{1}{q}}\left(J, \mathbb{R}^{+}\right)} \\
& \leq \frac{M(1+\|\psi\|+r)}{\Gamma(\alpha)} \lim _{\lambda \rightarrow 0}\left[\int_{0}^{t}\left[(t-s)^{\bar{\omega}}-(t+\lambda-s)^{\bar{\sigma}}\right] \mathrm{d} s\right]^{1-q}\|\varphi\|_{L^{\frac{\bar{q}}{(}}\left(J, \mathbb{R}^{+}\right)} \\
& \leq \frac{M(1+\|\psi\|+r)}{\Gamma(\alpha)} \lim _{\lambda \rightarrow 0}\left[\frac{1}{\omega+1}\left[t^{\bar{\omega}+1}+\lambda^{\bar{\omega}+1}-(t+\lambda)^{\overline{\bar{\omega}+1}}\right]\right]^{1-q}\|\varphi\|_{L^{\frac{1}{q}}\left(J, \mathbb{R}^{+}\right)}=0,
\end{aligned}
$$


independently of $x \in B_{r}\left(x^{*}\right)$.

For $G_{4}$, by using $\left(\mathrm{H}_{1}\right)$ and the Lebesgue dominated convergence theorem, we get

$$
\begin{aligned}
\lim _{\lambda \rightarrow 0} G_{4} & \leq \lim _{\lambda \rightarrow 0}\left\|\int_{0}^{t}(t-s)^{\alpha-1}\left[k_{2}(t+\lambda-s)-k_{2}(t-s)\right] f(s) \mathrm{d} s\right\| \\
& \leq \lim _{\lambda \rightarrow 0} \sup _{s \in[0, t]}\left\|K_{2}(t+\lambda-s)-K_{2}(t-s)\right\| \int_{0}^{t}(t-s)^{\alpha-1}\|f(s)\| \mathrm{d} s .
\end{aligned}
$$

By the uniform continuity of $K_{2}(t),(t>0)$, we conclude that $\lim _{\lambda \rightarrow 0} G_{4}=0$, independently of $x \in B_{r}\left(x^{*}\right)$.

$$
\begin{aligned}
\left\|y^{*}(t+\lambda)-y^{*}(t)\right\|= & \|y(t+\lambda)-y(t)\| \leq\left\|K_{1}(t+\lambda)(\psi(0)-g(x))-K_{1}(t)(\psi(0)-g(x))\right\| \\
& +\sum_{k=1}^{k=i}\left\|K_{1}\left(t+\lambda-t_{k}\right) I_{k}\left(x\left(t_{k}^{-}\right)\right)-K_{1}\left(t-t_{k}\right) I_{k}\left(x\left(t_{k}^{-}\right)\right)\right\| \\
& +\left\|\int_{0}^{t+\lambda}(t+\lambda-s)^{\alpha-1} k_{2}(t+\lambda-s) f(s) \mathrm{d} s-\int_{0}^{t}(t-s)^{\alpha-1} k_{2}(t-s) f(s) \mathrm{d} s\right\| .
\end{aligned}
$$

Arguing as in the first case we get

$$
\lim _{\lambda \rightarrow 0}\|y(t+\lambda)-y(t)\|=0 .
$$

Case 4. When $t=t_{i}, i=1, \cdots, m$, let $\lambda>0$ be such that $t_{i}+\lambda \in J_{i}$ and $\sigma>0$ such that

$$
t_{i}<\sigma<t_{i}+\lambda \leq t_{i+1} \text {, then we have }
$$

$$
\left\|y^{*}\left(t_{i}+\lambda\right)-y^{*}\left(t_{i}\right)\right\|=\lim _{\sigma \rightarrow t_{i}^{+}}\left\|y\left(t_{i}+\lambda\right)-y(\sigma)\right\|
$$

According the definition of $G$ we get

$$
\begin{aligned}
\left\|y\left(t_{i}+\lambda\right)-y(\sigma)\right\| \leq & \left\|k_{1}\left(t_{i}+\lambda\right)(\psi(0)-g(x))-k_{1}(\sigma)(\psi(0)-g(x))\right\| \\
& +\sum_{k=1}^{k=1}\left\|K_{1}\left(t_{i}+\lambda-t_{k}\right) I_{k}\left(x\left(t_{k}^{-}\right)\right)-K_{1}\left(\sigma-t_{k}\right) I_{k}\left(x\left(t_{k}^{-}\right)\right)\right\| \\
& +\left\|\int_{0}^{t_{i}+\lambda}\left(t_{i}+\lambda-s\right)^{\alpha-1} k_{2}\left(t_{i}+\lambda-s\right) f(s) \mathrm{d} s-\int_{0}^{\sigma}(\sigma-s)^{\alpha-1} k_{2}(\sigma-s) f(s) \mathrm{d} s\right\| .
\end{aligned}
$$

Arguing as in the first case we can see that

$$
\lim _{\substack{\lambda \rightarrow 0 \\ \sigma \rightarrow t_{i}^{+}}}\left\|y\left(t_{i}+\lambda\right)-y(\sigma)\right\|=0 .
$$

From (3.9) $\rightarrow$ (3.15) we conclude that $B_{\overline{J_{i}}}$ is equicontinuous for every $i=0,1, \cdots, m$.

Step 4. Our aim in this step is to show that for any $t \in[-r, b]$, the set

$$
\Omega(t)=\left\{y(t:): y \in G(x), x \in B_{r}\left(x^{*}\right)\right\}
$$

is relatively compact in $E$.

Let us introduce the following maps:

$$
G_{1}, G_{2}: \mathcal{H} \rightarrow \mathcal{H}, G_{3}: \mathcal{H} \rightarrow 2^{\mathcal{H}},
$$

where

$$
G_{1}(x)(t)= \begin{cases}\psi(t)-g(x), & t \in[-r, 0], \\ 0, & t \in(0, b),\end{cases}
$$

$$
G_{2}(x)(t)= \begin{cases}0, & t \in\left[-r, t_{1}\right], \\ \sum_{k=1}^{k=i} K_{1}\left(t-t_{k}\right) I_{k}\left(x\left(t_{k}^{-}\right)\right), & t \in J_{i}, i=1,2, \cdots, m,\end{cases}
$$

and $y \in G_{3}(x)$ if and only if

$$
y(t)= \begin{cases}0, & t \in[-r, 0], \\ K_{1}(t)(\psi(0)-g(x))+\int_{0}^{t}(t-s)^{\alpha-1} K_{2}(t-s) f_{y}(s) \mathrm{d} s, & t \in J-\left\{t_{1}, \cdots, t_{m}\right\} .\end{cases}
$$

where $f_{y} \in S_{F(\cdot, \tau(\cdot) x)}$. Obviously, $G=G_{1}+G_{2}+G_{3}$.

Because $B_{r}\left(x^{*}\right)$ is a bounded subset in $\mathcal{H}$ and $g$ 
is compact, the set

$$
\Omega_{1}(t)=\left\{G_{1}(x)(t): x \in B_{r}\left(x^{*}\right)\right\}
$$

is relatively compact in $E$. Also, since the functions $I_{k}, k=1,2, \cdots, m$ are compact, the set

$$
\begin{aligned}
& \Omega_{2}(t)=\left\{G_{2}(x)(t): x \in B_{r}\left(x^{*}\right)\right\} \quad \text { arbitrary } h \in(0, t) \text { and } \lambda \in(0,1) \text {, we define } \\
& y_{h, \lambda}(t)=\int_{\lambda}^{\infty} \zeta_{\alpha}(\theta) T\left(t^{\alpha} \theta\right)[\psi(0)-g(x)] \mathrm{d} \theta+\alpha \int_{0}^{t-h}(t-s)^{\alpha-1} \int_{\lambda}^{\infty} \theta \zeta_{\alpha}(\theta) T\left((t-s)^{\alpha} \theta\right) f_{y}(s) \mathrm{d} \theta \mathrm{d} s .
\end{aligned}
$$

Note that we can rewrite $y_{h, \lambda}(t)$ in the form

$$
\begin{aligned}
y_{h, \lambda}(t)= & T\left(h^{\alpha} \lambda\right) \int_{\lambda}^{\infty} \zeta_{\alpha}(\theta) T\left(t^{\alpha} \theta-h^{\alpha} \lambda\right)[\psi(0)-g(x)] \mathrm{d} \theta \\
& +T\left(h^{\alpha} \lambda\right) \int_{0}^{t-h}(t-s)^{\alpha-1}\left(\alpha \int_{\lambda}^{\infty} \theta \zeta_{\alpha}(\theta) T\left((t-s)^{\alpha} \theta-h^{\alpha} \lambda\right) \mathrm{d} \theta\right) f_{y}(s) \mathrm{d} s .
\end{aligned}
$$

Since the operator $T(t), t>0$ is compact and $g$ is compact on $\mathcal{H}$, the set

$$
\left\{y_{h, \lambda}(t): y \in G_{3}(x), x \in B_{r}\left(x^{*}\right)\right\}
$$

is relatively compact in $E$. Moreover, by using $\left(\mathrm{H}_{3}\right)$ and $\left(\mathrm{H}_{4}\right)$ we get

$$
\begin{aligned}
& \left\|y(t)-y_{h, \lambda}(t)\right\| \leq\left\|\int_{0}^{\lambda} \zeta_{\alpha}(\theta) T\left(t^{\alpha} \theta\right)[\psi(0)-g(x)] \mathrm{d} \theta\right\| \\
& +\alpha\left\|\int_{0}^{t} \int_{0}^{\infty} \theta(t-s)^{\alpha-1} \zeta_{\alpha}(\theta) T\left((t-s)^{\alpha} \theta\right) f_{y}(s) \mathrm{d} \theta \mathrm{d} s-\int_{0}^{t-h} \int_{\lambda}^{\infty} \theta(t-s)^{\alpha-1} \zeta_{\alpha}(\theta) T\left((t-s)^{\alpha} \theta\right) f_{y}(s) \mathrm{d} \theta \mathrm{d} s\right\| \\
& \leq M(\|\psi(0)\|+\|g(x)\|) \int_{0}^{\lambda} \zeta_{\alpha}(\theta) \mathrm{d} \theta+\alpha \| \int_{0}^{t} \int_{\lambda}^{\infty} \theta(t-s)^{\alpha-1} \zeta_{\alpha}(\theta) T\left((t-s)^{\alpha} \theta\right) f_{y}(s) \mathrm{d} \theta \mathrm{d} s \\
& +\int_{0}^{t} \int_{0}^{\lambda} \theta(t-s)^{\alpha-1} \zeta_{\alpha}(\theta) T\left((t-s)^{\alpha} \theta\right) f_{y}(s) \mathrm{d} \theta \mathrm{d} s-\int_{0}^{t-h} \int_{\lambda}^{\infty} \theta(t-s)^{\alpha-1} \zeta_{\alpha}(\theta) T\left((t-s)^{\alpha} \theta\right) f_{y}(s) \mathrm{d} \theta \mathrm{d} s \| \\
& \leq M(\|\psi(0)\|+a\|x\|+d) \int_{0}^{\lambda} \zeta_{\alpha}(\theta) \mathrm{d} \theta+\alpha\left\|\int_{t-h}^{t} \int_{\lambda}^{\infty} \theta(t-s)^{\alpha-1} \zeta_{\alpha}(\theta) T\left((t-s)^{\alpha} \theta\right) f_{y}(s) \mathrm{d} \theta \mathrm{d} s\right\| \\
& +\alpha\left\|\int_{0}^{t} \int_{0}^{\lambda} \theta(t-s)^{\alpha-1} \zeta_{\alpha}(\theta) T\left((t-s)^{\alpha} \theta\right) f_{y}(s) \mathrm{d} \theta \mathrm{d} s\right\| \\
& \leq M(\|\psi(0)\|+a r+a\|\psi\|+d) \int_{0}^{\lambda} \zeta_{\alpha}(\theta) \mathrm{d} \theta+M \alpha \int_{t-h}^{t} \int_{\lambda}^{\infty} \theta(t-s)^{\alpha-1} \zeta_{\alpha}(\theta) \varphi(s)(1+\|x\|) \mathrm{d} \theta \mathrm{d} s \\
& +M \alpha \int_{0}^{t} \int_{0}^{\lambda} \theta(t-s)^{\alpha-1} \zeta_{\alpha}(\theta) \varphi(s)(1+\|x\|) \mathrm{d} \theta \mathrm{d} s \\
& \leq M(\|\psi(0)\|+a r+a\|\psi\|+d) \int_{0}^{\lambda} \zeta_{\alpha}(\theta) \mathrm{d} \theta+M \alpha(1+r+\|\psi\|) \int_{\lambda}^{\infty} \theta \zeta_{\alpha}(\theta) \mathrm{d} \theta \int_{t-h}^{t}(t-s)^{\alpha-1} \varphi(s) \mathrm{d} s \\
& +M \alpha(1+r+\|\psi\|) \int_{0}^{\lambda} \theta \zeta_{\alpha}(\theta) \mathrm{d} \theta \int_{0}^{t}(t-s)^{\alpha-1} \varphi(s) \mathrm{d} s .
\end{aligned}
$$

Using Hölder’s inequality to get

$$
\begin{aligned}
& \left\|y(t)-y_{h, \lambda}(t)\right\| \leq M(\|\psi(0)\|+a r+a\|\psi\|+d) \int_{0}^{\lambda} \zeta_{\alpha}(\theta) \mathrm{d} \theta \\
& +M \alpha(1+r+\|\psi\|)\|\varphi\|_{L^{q}\left(J, \mathbb{R}^{+}\right)}^{\frac{1}{(\varpi+1)^{1-q}}} \int_{\lambda}^{\infty} \theta \zeta_{\alpha}(\theta) \mathrm{d} \theta+M \alpha(1+r+\|\psi\|)\|\varphi\|_{L^{\frac{1}{q}}\left(J, \mathbb{R}^{+}\right)} \frac{b^{\alpha-q}}{(\varpi+1)^{1-q}} \int_{0}^{\lambda} \theta \zeta_{q}(\theta) \mathrm{d} \theta .
\end{aligned}
$$

Obviously, by Lemma 4(2), the right hand side of the previous inequality tend to zero as $\lambda, h \rightarrow 0$. Hence,

there exists a relatively compact set that can be arbitrary close to the set $\Omega_{3}(t), t \in(0, b]$. Hence, this set is rela- 
tively compact in $E$. Hence, $\Omega(t), t \in[-r, b]$ is relatively compact

As a consequence of Steps 3 and 4 with Arzela-Ascoli theorem we conclude that, $B$ is relatively compact.

Step 5. $G$ has a closed graph on $B$.
Let $x_{n} \in B_{r}\left(x^{*}\right), x_{n} \rightarrow x$ in $\mathcal{H}$ and $y_{n} \in G\left(x_{n}\right), \forall n \geq 1$ with $y_{n} \rightarrow y$ in $B$. We will show that $y \in G(x)$. By recalling the definition of $G$, for any $n \geq 1$ there exists $f_{n} \in S_{F\left(\cdot, \tau(\cdot) x_{n}\right)}$ such that

$$
y_{n}(t)=\left\{\begin{array}{l}
\psi(t)-g\left(x_{n}\right), t \in[-r, 0], \\
K_{1}(t)\left(\psi(0)-g\left(x_{n}\right)\right)+\int_{0}^{t}(t-s)^{\alpha-1} K_{2}(t-s) f_{n}(s) \mathrm{d} s, t \in J_{0}, \\
K_{1}(t)\left(\psi(0)-g\left(x_{n}\right)\right)+\sum_{k=1}^{k=i} K_{1}\left(t-t_{k}\right) I_{k}\left(x_{n}\left(t_{k}^{-}\right)\right)+\int_{0}^{t}(t-s)^{\alpha-1} K_{2}(t-s) f_{n}(s) \mathrm{d} s, t \in J_{i}, i=1,2, \cdots, m .
\end{array}\right.
$$

Let us show that the sequence $\left(f_{n}\right)_{n \geq 1}$ is semicompact. From the uniform convergence of $x_{n}$ towards $x$, for any $t \in J$

$$
\lim _{n \rightarrow \infty}\left\|\tau(t) x_{n}-\tau(t) x\right\|_{\Phi}=0
$$

Moreover $F(t,$.$) is upper semicontinuous with$ compact values, then for every $\epsilon>0$, there exists a natural number $n_{0}(\epsilon)$ such that for every $n \geq n_{0}$

$$
\begin{aligned}
& f_{n}(t) \in F\left(t, \tau(t) x_{n}\right) \\
& \subseteq F(t, \tau(t) x)+\epsilon B(0,1), \text { a.e. } t \in J,
\end{aligned}
$$

where $B(0,1)=\{z \in E:\|z\| \leq 1\}$. Then, the compactness of $F(t, \tau(t) x)$ implies that the set $\left\{f_{n}(t): n \geq 1\right\}$ is relatively compact for a.e. . In addition, assumption $\left(\mathrm{H}_{3}\right)$ implies

$$
\begin{aligned}
\left\|f_{n}(t)\right\| & \leq \varphi(t)\left\|1+\tau(t) x_{n}(0)\right\| \\
& \leq \varphi(t)\left(1+\left\|x_{n}\right\|\right) \\
& \leq \varphi(t)(1+r+\|\psi\|), \text { a.e. } t \in J .
\end{aligned}
$$

Then, by Lemma 2, $\left\{f_{n}, n \geq 1\right\}$ is semicompact, hence weakly compact. Arguing as in Step 1 from Mazur's theorem, there is a sequence $\left(z_{n}\right), n \geq 1$ such that

$$
\left\{z_{n}(t): n \geq 1\right\} \subseteq \overline{\operatorname{Conv}}\left\{f_{n}(t): n \geq 1\right\} ; t \in J
$$

and $z_{n}$ converges strongly to $f \in L^{1}(J, E)$. Since, the values of $F$ are convex, $z_{n} \in S_{F\left(., \tau(.) x_{n}\right)}$ and hence, $f \in S_{F(., \tau(.) x)}$. By passing to the limit in (3.16), with taking into account that $g$ is continuous, we obtain

$$
y(t)=\left\{\begin{array}{l}
\psi(t)-g(x), t \in[-r, 0] \\
K_{1}(t)(\psi(0)-g(x))+\int_{0}^{t}(t-s)^{\alpha-1} K_{2}(t-s) f(s) \mathrm{d} s, t \in J_{0}, \\
K_{1}(t)(\psi(0)-g(x))+\sum_{k=1}^{k=i} K_{1}\left(t-t_{k}\right) I_{k}\left(x\left(t_{k}^{-}\right)\right)+\int_{0}^{t}(t-s)^{\alpha-1} K_{2}(t-s) f(s) \mathrm{d} s, t \in J_{i}, i=1,2, \cdots, m .
\end{array}\right.
$$

This proves that the graph of $G$ is closed.

Now, as a consequence of Step 1 to Step 5, we conclude that the multifunction of $G$ is a compact multivalued function, u.s.c with convex compact values. By applying Theorem 1, we can deduce that $G$ has a fixed point $x$ which is a mild solution of Problem (1.1).

In the following Theorems we give another version for an existence result for (1.1).

Theorem 5 Let $F: J \times \Phi \rightarrow P_{c k}(E)$ be a multifunction, $A$ is the infinitesimal generator of a $C_{0}$-semigroup $\{T(t): t \geq 0\}$, and $g: \mathcal{H} \rightarrow E$. We suppose the following assumptions:

$\left(\mathrm{H}_{6}\right)$ For every $x \in \Phi, t \rightarrow F(t, x)$ is measurable.
$\left(\mathrm{H}_{7}\right)$ There is a function $\varsigma \in L^{\frac{1}{q}}\left(J, \mathbb{R}^{+}\right),(0<q<\alpha)$ such that

For every $x, y \in \Phi$

$$
\begin{aligned}
& h(F(t, x), F(t, y)) \leq \varsigma(t)\|x(0)-y(0)\|, \\
& \text { for a.e. } t \in J, \\
& \operatorname{Sup}\{\|z\|: x \in F(t, 0)\} \leq \varsigma(t), \text { for a.e. } t \in J .
\end{aligned}
$$

$\left(\mathrm{H}_{8}\right)$ There is a positive constant $a$ such that

$$
\|g(z)-g(w)\| \leq a\|z-w\|, \text { for all } z, w \in \mathcal{H} .
$$

$\left(\mathrm{H}_{9}\right)$ For each $i=1,2, \cdots, m$, there is $\xi_{i}>0$ such that 


$$
\left\|I_{i}(x)-I_{i}(y)\right\| \leq \xi_{i}\|x-y\|_{\mathcal{H}}, \text { for all } x, y \in E .
$$

$\left(\mathrm{H}_{10}\right)$

$$
a+M\left[a+\xi+\frac{\gamma}{\Gamma(\alpha)}\right]<1
$$

where $\gamma=\frac{b^{\alpha-q}}{(\bar{\omega}+1)^{1-q}}\|\varsigma\|_{L^{\frac{1}{q}}\left(J, \mathbb{R}^{+}\right)}, \quad \bar{\omega}=\frac{\alpha-1}{1-q}$ and $\xi=\sum_{i=1}^{i=m} \xi_{i}$.

Then (1.1) has a mild solution.

Proof. For $x \in \mathcal{H}$, set

$$
S_{F(., \tau(\cdot) x)}^{1}=\left\{f \in L^{1}(J, E): f(t) \in F(t, \tau(t) x) \text { for a.e. } t \in J\right\} .
$$

By Lemma 1, $\left(\mathrm{H}_{6}\right)$ and $\left(\mathrm{H}_{7}\right), F(., \tau() x$.$) is meas-$ urable. Since its values are closed, it has a measurable selection (see [29], Theorem 8.1.3) which, by hypothesis $\left(\mathrm{H}_{7}\right)$, belongs to $L^{1}(J, E)$. Thus $S_{F(\cdot, \tau(\cdot) x)}^{1}$ is non- empty. Let us transform the problem into a fixed point problem. Consider the multifunction map, $R: \mathcal{H} \rightarrow 2^{\mathcal{H}}$ defined as follows: for $x \in \mathcal{H}, R(x)$ is the set of all functions $y \in R(x)$, such that for each $i=1,2, \cdots, m$,

$$
y(t)=\left\{\begin{array}{l}
\psi(t)-g(x), t \in[-r, 0], \\
K_{1}(t)(\psi(0)-g(x))+\int_{0}^{t}(t-s)^{\alpha-1} K_{2}(t-s) f(s) \mathrm{d} s, t \in J_{0}, \\
K_{1}(t)(\psi(0)-g(x))+\sum_{k=1}^{k=i} K_{1}\left(t-t_{k}\right) I_{k}\left(x\left(t_{k}^{-}\right)\right)+\int_{0}^{t}(t-s)^{\alpha-1} K_{2}(t-s) f(s) \mathrm{d} s, t \in J_{i}, i=1, \cdots, m .
\end{array}\right.
$$

where $f \in S_{F(\cdot . \tau(\cdot) x)}^{1}$. It is easy to see that any fixed point for $R$ is a mild solution for (1.1). So, we shall show that $R$ satisfies the assumptions of Theorem 2. The proof will be given in two steps.

Step1. The values of $R$ are nonempty and closed.

Since $S_{F(., \tau(\cdot) x)}^{1}$ is non-empty, the values of $R$ are non-empty. In order to prove the values of $R$ are closed, let $x \in \mathcal{H}$ and $\left(y_{n}\right), n \geq 1$ be a sequence in $R(x)$ such that $y_{n} \rightarrow y$ in $\mathcal{H}$. Then, according to the definition of $R$, there is a sequence $\left(f_{n}\right), n \geq 1$ in $S_{F(., \tau(\cdot) x)}^{1}$ such that for any $t \in J_{i}, i=0,1, \cdots, m$

$$
y_{n}(t)=\left\{\begin{array}{l}
\psi(t)-g(x), t \in[-r, 0] \\
K_{1}(t)(\psi(0)-g(x))+\int_{0}^{t}(t-s)^{\alpha-1} K_{2}(t-s) f_{n}(s) \mathrm{d} s, t \in J_{0}, \\
K_{1}(t)(\psi(0)-g(x))+\sum_{k=1}^{k=i} K_{1}\left(t-t_{k}\right) I_{k}\left(x\left(t_{k}^{-}\right)\right)+\int_{0}^{t}(t-s)^{\alpha-1} K_{2}(t-s) f_{n}(s) \mathrm{d} s, t \in J_{i}, i=1,2, \cdots, m .
\end{array}\right.
$$

Since $F(t, 0)$ is closed, for any $t \in J$, there is $v(t) \in F(t, 0)$ such that $\|v(t)\|=d(0, F(t, 0))$. In view of $\left(\mathrm{H}_{9}\right)$, for every $n \geq 1$, and for a.e. $t \in J$

$$
\begin{aligned}
\left\|f_{n}(t)\right\| & \leq\|v(t)\|+d\left(f_{n}(t), F(t, 0)\right) \\
& =d(0, F(t, 0))+d\left(f_{n}(t), F(t, 0)\right) \\
& \leq \varsigma(t)+H(F(t, \tau(t) x), F(t, 0)) \\
& \leq \varsigma(t)+\varsigma(t)\|\tau(t) x(0)\|_{E} \\
& =\varsigma(t)\left(1+\|x(t)\|_{E}\right) \leq \varsigma(t)\left(1+\|x\|_{\mathcal{H}}\right) .
\end{aligned}
$$

This show that the set $\left\{f_{n}: n \geq 1\right\}$ is integrably bounded. Using the fact that $F$ has compact values, the set $\left\{f_{n}(t): n \geq 1\right\}$ is relativity compact in $E$ for a.e. $t \in J$. Therefore, the set $\left\{f_{n}: n \geq 1\right\}$ is semi-compact and in $L^{1}(J, E)$. Then, by Lemma 2, it is weakly compact. So, we may pass to a subsequence if necessary to get that $f_{n}$ converges weakly to a function

$f \in L^{1}(J, E)$. From Mazur's theorem, there is a sequence $\left(z_{n}\right), n \geq 1$ such that

$$
\left\{z_{n}(t): n \geq 1\right\} \subseteq \overline{\operatorname{Conv}}\left\{f_{n}(t): n \geq 1\right\} ; t \in J
$$

and $z_{n}$ converges strongly to $f$. Since, the values of $F$ are convex, $z_{n} \in S_{F(., \tau(.) x)}^{1}$ and hence, by the compactness of $F(., \tau() x),. \quad f \in S_{F(., \tau(.) x)}^{1} . \quad$ Note that for every $t, s \in J, s \in(0, t]$ and for every $n \geq 1$,

$$
\begin{aligned}
& |t-s|^{\alpha-1}\left\|K_{2}(t-s) f_{n}(s)\right\| \\
& \leq|t-s|^{\alpha-1} \frac{M}{\Gamma(\alpha)} \varsigma(t)\left(1+\|x\|_{\mathcal{H}}\right) \in L^{1}\left((0, t], R^{+}\right) .
\end{aligned}
$$


Therefore, by means of the Lebesgue dominated convergence Theorem and the continuity of $K_{2}(t), t \geq 0$

we obtain from (3.17)

$$
\lim _{n \rightarrow \infty} y_{n}(t)=\left\{\begin{array}{l}
\psi(t)-g(x), t \in[-r, 0] \\
K_{1}(t)(\psi(0)-g(x))+\int_{0}^{t}(t-s)^{\alpha-1} K_{2}(t-s) f(s) \mathrm{d} s, t \in J_{0}, \\
K_{1}(t)(\psi(0)-g(x))+\sum_{k=1}^{k=1} K_{1}\left(t-t_{k}\right) I_{k}\left(x\left(t_{k}^{-}\right)\right)+\int_{0}^{t}(t-s)^{\alpha-1} K_{2}(t-s) f(s) \mathrm{d} s, t \in J_{i}, i=1,2, \cdots, m .
\end{array}\right.
$$

So, $y \in R(x)$.

Step 2. $R$ is contraction. Let $x_{2}, x_{1} \in \mathcal{H}$ and $y_{1} \in R\left(x_{1}\right)$. Then, there is $f \in S_{F\left(., \tau(\cdot) x_{1}\right)}^{1}$ such that for

$$
y_{1}(t)=\left\{\begin{array}{l}
\psi(t)-g\left(x_{1}\right), t \in[-r, 0] \\
K_{1}(t)\left(\psi(0)-g\left(x_{1}\right)\right)+\int_{0}^{t}(t-s)^{\alpha-1} K_{2}(t-s) f(s) \mathrm{d} s, t \in J_{0}, \\
K_{1}(t)\left(\psi(0)-g\left(x_{1}\right)\right)+\sum_{k=1}^{k=i} K_{1}\left(t-t_{k}\right) I_{k}\left(x\left(t_{k}^{-}\right)\right)+\int_{0}^{t}(t-s)^{\alpha-1} K_{2}(t-s) f(s) \mathrm{d} s, t \in J_{i}, i=1,2, \cdots, m .
\end{array}\right.
$$

Consider the multifunction $Z: J \rightarrow 2^{E}$ defined by

$$
Z(t)=\left\{u \in E:\|f(t)-u\| \leq \varsigma(t)\left\|x_{1}(t)-x_{2}(t)\right\|\right\} .
$$

For each $t \in J, Z(t)$ is nonempty. Indeed, let $t \in J$, from $\left(\mathrm{H}_{7}\right)$, we have

$$
\begin{aligned}
& h\left(F\left(t, \tau(t) x_{1}\right), F\left(t, \tau(t) x_{2}\right)\right) \\
& \leq \varsigma(t)\left\|\tau(t) x_{1}(0)-\tau(t) x_{2}(0)\right\| \\
& =\varsigma(t)\left\|x_{1}(t)-x_{2}(t)\right\| .
\end{aligned}
$$

Hence, there exists $u_{t} \in F\left(t, x_{2}(t)\right)$ such that

$$
\left\|u_{t}-f(t)\right\| \leq \varsigma(t)\left\|x_{1}(t)-x_{2}(t)\right\| .
$$

Since the functions $f, \varsigma, x_{1}, x_{2}$ are measurable, Proposition III.4 in [30], tells us that the multifunction $V: t \rightarrow Z(t) \cap F\left(t, \tau(t) x_{2}\right)$ is measurable. Because its values are nonempty and closed there is $h \in S_{F\left(t, \tau(t) x_{2}\right)}^{1}$ with

$$
\begin{aligned}
& \|h(t)-f(t)\| \leq \varsigma(t)\left\|x_{1}(t)-x_{2}(t)\right\| \\
& \leq \varsigma(t)\left\|x_{1}-x_{2}\right\|_{\mathcal{H}}, \text { a.e. } t \in J .
\end{aligned}
$$

Let us define

$$
y_{2}(t)=\left\{\begin{array}{l}
\psi(t)-g\left(x_{2}\right), t \in[-r, 0] \\
K_{1}(t)\left(\psi(0)-g\left(x_{2}\right)\right)+\int_{0}^{t}(t-s)^{\alpha-1} K_{2}(t-s) h(s) \mathrm{d} s, t \in J_{0}, \\
K_{1}(t)\left(\psi(0)-g\left(x_{2}\right)\right)+\sum_{k=1}^{k=i} K_{1}\left(t-t_{k}\right) I_{k}\left(x\left(t_{k}^{-}\right)\right)+\int_{0}^{t}(t-s)^{\alpha-1} K_{2}(t-s) h(s) \mathrm{d} s, t \in J_{i}, i=1,2, \cdots, m .
\end{array}\right.
$$

Obviously, $y_{2} \in R\left(x_{2}\right)$ and if $t \in[-r, 0]$, then by $\left(\mathrm{H}_{8}\right)$

$$
\left\|y_{2}(t)-y_{1}(t)\right\| \leq a\left\|x_{1}-x_{2}\right\|_{\mathcal{H}} .
$$

If $t \in J_{0}$, we get from $(3.18) \rightarrow(3.20)$ and $\left(\mathrm{H}_{8}\right)$

$$
\begin{aligned}
\left\|y_{2}(t)-y_{1}(t)\right\| & \leq M\left\|g\left(x_{1}\right)-g\left(x_{2}\right)\right\|+\frac{M}{\Gamma(\alpha)} \int_{0}^{t}(t-s)^{\alpha-1}\|h(s)-f(s)\| \mathrm{d} s \\
& \leq M a\left\|x_{1}-x_{2}\right\|_{\mathcal{H}}+\frac{M}{\Gamma(\alpha)}\left\|x_{1}-x_{2}\right\|_{\mathcal{H}} \int_{0}^{t}(t-s)^{\alpha-1} \varsigma(s) \mathrm{d} s \\
& \left.\leq M a\left\|x_{1}-x_{2}\right\|_{\mathcal{H}}+\frac{M}{\Gamma(\alpha)}\left\|x_{1}-x_{2}\right\|_{\mathcal{H}}\|s\|_{L^{q}}^{\frac{1}{q} J, \mathbb{R}^{+}}\right)\left(\int_{0}^{t}(t-s)^{\frac{\alpha-1}{1-q}} \mathrm{~d} s\right)^{1-q} \leq\left\|x_{1}-x_{2}\right\|_{\mathcal{H}}\left(M a+\frac{M}{\Gamma(\alpha)} \gamma\right)
\end{aligned}
$$


Similarly, if $t \in J_{i}, i=1, \cdots, m$, we get from $(3.18) \rightarrow(3.20),\left(\mathrm{H}_{8}\right)$ and $\left(\mathrm{H}_{9}\right)$

$$
\left\|y_{2}(t)-y_{1}(t)\right\| \leq M(a+\xi)\left\|x_{1}-x_{2}\right\|_{\mathcal{H}}+\frac{M}{\Gamma(\alpha)} \int_{0}^{t}(t-s)^{\alpha-1}\|h(s)-f(s)\| \mathrm{d} s \leq\left\|x_{1}-x_{2}\right\|_{\mathcal{H}} M\left[a+\xi+\frac{\gamma}{\Gamma(\alpha)}\right] .
$$

By interchanging the role of $y_{2}$ and $y_{1}$, we obtain from (3.21), (3.22) and $\left(\mathrm{H}_{10}\right)$

$$
\left\|R\left(x_{2}\right)-R\left(x_{1}\right)\right\| \leq\left\|x_{1}-x_{2}\right\|_{\mathcal{H}} .
$$

Therefore, $R$ is contraction and thus by Theorem 2 $R$ has a fixed point which is a mild solution for (1.1).

\subsection{Nonconvex Case}

In the following Theorem we give nonconvex version for Theorem 4. Our hypothesis on the orient field is the following:

$\left(\mathrm{H}_{11}\right) \quad F: J \times \Phi \rightarrow P_{c l}(E)$ is a multifunction such that
1) $(t, x) \rightarrow F(t, x)$ is graph measurable and $x \rightarrow F(t, x)$ is lower semicontinuous.

2) There exists a function $\varphi \in L^{\frac{1}{q}}\left(J, \mathbb{R}^{+}\right), \quad 0<q<\alpha$ such that for any $x \in \Phi$

$$
\|F(t, x)\| \leq \varphi(t) \text {, a.e. } t \in J \text {. }
$$

Theorem 6 If the hypotheses $\left(\mathrm{H}_{1}\right),\left(\mathrm{H}_{4}\right)$, $\left(\mathrm{H}_{5}\right)$ and $\left(\mathrm{H}_{11}\right)$, then the problem (1.1) has a mild solution provided that there is $r>0$ such that the condition (3.4) is satisfied.

Proof. Consider the multivalued Nemitsky operator $N: \mathcal{H} \rightarrow 2^{L^{1}(J, E)}$, defined by

$$
N(x)=S_{F(., \tau(\cdot) x)}^{1}=\left\{f \in L^{1}(J, E): f(t) \in F(t, \tau(t) x) \text {, a.e. } t \in J\right\} .
$$

We shall prove that $N$ has a nonempty closed decomposable value and l.s.c. Since $F$ has closed values, $S_{F}^{1}$ is closed ([37]). Because $F$ is integrably bounded, $S_{F}^{1}$ is nonempty (see, Theorem 3.2 [37]). It is readily verified, $S_{F}^{1}$ is decomposable. To check the lower semi-continuity of $N$, we need to show that, for every $u \in L^{1}(J, E), \quad x \rightarrow d(u, N(x))$ is upper semicontinuous. To this end from Theorem 2.2 [37], we have

$$
\begin{aligned}
d(u, N(x)) & =\inf _{v \in N(x)}\|u-v\|_{L^{1}}=\inf _{v(t) \in F(t, \tau(t) x)} \int_{0}^{b}\|u(t)-v(t)\| \mathrm{d} t \\
& =\int_{0}^{b} \inf _{z(t) \in F(t, \tau(t) x)}\|u(t)-z(t)\| \mathrm{d} t=\int_{0}^{b} d(u(t), F(t, \tau(t) x)) \mathrm{d} t .
\end{aligned}
$$

We shall show that, for any $\lambda \geq 0$, the set

$$
u_{\lambda}=\{x \in \mathcal{H}: d(u, N(x)) \geq \lambda\}
$$

is closed. For this purpose, let $\left\{x_{n}\right\} \subseteq u_{\lambda}$ and assume

that $x_{n} \rightarrow x$ in $\mathcal{H}$. Then, for all $t \in J, \quad x_{n}(t) \rightarrow x(t)$ in $E$. By virtue of $\left(\mathrm{H}_{11}\right)(1)$ the function $z \rightarrow d(u(t), F(t, z))$ is u.s.c. So, via the Fatou Lemma, and (3.23) we have

$$
\begin{aligned}
\lambda & \leq \limsup _{n \rightarrow \infty} d\left(u, N\left(x_{n}\right)\right)=\limsup _{n \rightarrow \infty} \int_{0}^{b} d\left(u(t), F\left(t, \tau(t) x_{n}\right)\right) \mathrm{d} t \\
& \leq \int_{0}^{b} \lim _{n \rightarrow \infty} \sup d\left(u(t), F\left(t, \tau(t) x_{n}\right)\right) \mathrm{d} t \leq \int_{0}^{b} d(u(t), F(t, \tau(t) x)) \mathrm{d} t=d(u, N(x)) .
\end{aligned}
$$

Therefore, $x \in u_{\lambda}$ and this proves the lower semicontinuity of $N$. This allows us to apply Theorem 3 of [38] and obtain a continuous map $Z: \mathcal{H} \rightarrow L^{1}(J, E)$ such

that $Z(x) \in N(x)$, for every $x \in \mathcal{H}$. Then, $Z(x)(s) \in F(s, \tau(s) x)$, a.e. $s \in J$.

Consider a map $\Lambda: \mathcal{H} \rightarrow \mathcal{H}$ defined by

$$
(\Lambda x)(t)=\left\{\begin{array}{l}
\psi(t)-g(x), t \in[-r, 0] \\
\left.K_{1}(t)(\psi(0)-g(x))+\int_{0}^{t}(t-s)^{\alpha-1} K_{2}(t-s)\right) Z(x)(s) \mathrm{d} s, t \in J_{0}, \\
\left.K_{1}(t)(\psi(0)-g(x))+\sum_{k=1}^{k=i} K_{1}\left(t-t_{k}\right) I_{k}\left(x\left(t_{k}^{-}\right)\right)+\int_{0}^{t}(t-s)^{\alpha-1} K_{2}(t-s)\right) Z(x)(s) \mathrm{d} s, t \in J_{i}, i=1,2, \cdots, m .
\end{array}\right.
$$


Arguing as in the proof of Theorem 4, we can show that $\Lambda$ satiesfies all the conditions of Theorem 3 (Schauder fixed point theorem). Thus, there is $x \in \mathcal{H}$ such that $x(t)=(\Lambda x)(t)$. This means that $x$ is a mild solution for (1.1).

Remark 4 The condition (3.4) will be satisfied if

$$
a+M(a+h+\eta)<1 .
$$

Indeed, condition (3.4) can be written as

$$
\frac{(M+1)[\|\psi\|(1+a)+d]+M h\|\psi\|+M \eta(1+\|\psi\|)}{1-[a+M(a+h+\eta)]} \leq r .
$$

\section{Conclusion}

In this paper, existence problems of nonlocal fractionalorder impulsive semi-linear differential inclusions with delay have been considered. We have been considered the case when the values of the orient field are convex as well as non-convex. Some sufficient conditions have been obtained, as pointed in the first section, theses conditions are strictly weaker than the most of the existing ones. In addition, our technique allows us to discuss some fractional differential inclusions with delay.

\section{REFERENCES}

[1] W. H. Glocke and T. F. Nonnemacher, "A Fractional CalCulus Approach of Self-Similar Protein Dynamics,” Biophysical Journal, Vol. 68, No. 1, 1995, pp. 46-53. doi:10.1016/S0006-3495(95)80157-8

[2] R. Hilfer, "Applications of Fractional Calculus in Physics,” World Scientific, Singapore City, 1999.

[3] A. A. Kilbas, H. M. Srivastava and J. J. Trujillo, "Theory and Applications of Fractional Differential Equations, in: North Holland Mathematics Studies, 204,” Elsevier Science, Publishers BV, Amsterdam, 2006.

[4] K. S. Miller and B. Ross, "An Introduction to the Fractional Calculus and Differential Equations,” John Wiley, New York, 1993.

[5] A. M. A. El-Sayed and A. G. Ibrahim, "Multivalued DifFerential Inclusions," Applied Mathematics and Computation, Vol. 68, No. 1, 1995, pp. 15-25. doi:10.1016/0096-3003(94)00080-N

[6] B. Ahmed, "Existence Results for Fractional Differential Inclusions with Separated Boundary Conditions,” Bulletin of the Korean Mathematical Society, Vol. 47, No. 4, 2010, pp. 805-813.

[7] R. P. Agarwal, M. Benchohra and B. A. Slimani, "Existence Results for Differential Equations with Fractional Order and Impulses,” Mem. Differential Equations, Math. Phys., Vol. 44, 2008, pp. 1-21.

[8] M. Belmekki and M. Benchohra, "Existence Results for Fractional Order Semilinear Functional Differential Equations with Nondense Domain," Nonlinear Analysis, Vol. 72, No. 2, 2010, pp. 925-932.

\section{doi:10.1016/j.na.2009.07.034}

[9] A. Ouahab, "Fractional Semilinear Differential Inclusions," Computer and Mathematics with Applications, Vol. 64, No. 10, 2012, pp. 3235-3252.

[10] J.-R. Wang, M. Feckan and Y. Zhou, "On the New Concept of Solutions and Existence Results for Impulsive Fractional Evolutions,” Dynamics of PDE, Vol. 8, No. 4, 2011, pp. 345-361.

[11] J. R. Wang and Y. Zhou, "Existence and Controllability Results for Fractional Semilinear Differential Inclusions," Nonlinear Analysis: Real World Applications, Vol. 12, No. 6, 2011, pp. 3642-3653. doi:10.1016/j.nonrwa.2011.06.021

[12] Z. Zhang and B. Liu, "Existence of Mild Solutions for Fractional Evolutions Equations,” Journal of Fractional Calculus and Applications, Vol. 2, No. 10, 2012, pp. 1-10.

[13] Y. Zhou and F. Jiao, "Nonlocal Cauchy Problem for Fractional Evolution Equations,” Nonlinear Analysis: RWA, Vol. 11, No. 5, 2010, pp. 4465-4475.

[14] Y. Zhou and F. Jiao, "Nonlocal Cauchy Problem for Fractional Natural Evolution Equations," Computer and Mathematics with Applications, Vol. 59, No. 3, 2010, pp. 10631077. doi:10.1016/j.camwa.2009.06.026

[15] Z. Agur, L. Cojocaru, G. Mazaur, R. M. Anderson and Y. L. Danon, "Pulse Mass Measles Vaccination across Age Shorts," Proceedings of the National Academy of Sciences of the United States of America, Vol. 90, No. 24, 1993, pp. 11698-11702. doi:10.1073/pnas.90.24.11698

[16] G. Ballinger and X. Liu, "Boundedness for Impulsive Delay Differential Equations and Applications in Populations Growth Models,” Nonlinear Analysis, Vol. 53, No. 7-8, 2003, pp. 1041-1062. doi:10.1016/S0362-546X(03)00041-5

[17] A. D. Onofrio, "On Pulse Vaccination Strategy in the SIR Epidemic Model with Vertical Transmission,” Applied Mathematics Letters, Vol. 18, No. 7, 2005, pp. 729-732. doi:10.1016/j.aml.2004.05.012

[18] M. Benchohra, J. Henderson and S. Ntouyas, "Impulsive Differential Equations and Inclusions,” Hindawi Publishing, New York, Egypt, 2007.

[19] J. M. Ball, "Initial Boundary Value Problems for an Extensible Beam,” Journal of Mathematical Analysis and Applications, Vol. 42, No. 1, 1973, pp. 16-90. doi:10.1016/0022-247X(73)90121-2

[20] W. E. Fitzgibbon, "Global Existence and Boundedness of Solutions to the Extensible Beam Equation," SIAM Journal on Mathematical Analysis, Vol. 13, No. 5, 1982, pp. 739-745. doi: $10.1137 / 0513050$

[21] R. A. Al-Omair and A. G. Ibrahim, "Existence of Mild Solutions of a Semilinear Evolution Differential Inclusions with Nonlocal Conditions,” EJDE, 2009, pp. 1-11.

[22] T. Cardinali and P. Rubbioni, "Impulsive Mild Solution for Semilinear Differential Inclusions with Nonlocal Conditions in Banach Spaces,” Nonlinear Analysis, Vol. 75, No. 2, 2012, pp. 871-879. doi:10.1016/j.na.2011.09.023

[23] Z. Fan, "Impulsive Problems for Semilinear Differential Equations with Nonlocal Conditions,” Nonlinear Analysis, Vol. 72, No. 2, 2010, pp. 1104-1109. 


$$
\text { doi:10.1016/j.na.2009.07.049 }
$$

[24] G. M. Mophou, "Existence and Uniqueness of Mild Solution to Impulsive Fractional Differential Equations," Nonlinear Analysis: Theory, Methods \& Applications, Vol. 72, No. 3-4, 2010, pp. 1604-1615.

[25] O. K. Jaradat, A. Al-Omari and S. Momani, "Existence of the Mild Solution for Fractional Semi-Linear Initial Value problems," Nonlinear Analysis: Theory, Methods \& Applications, Vol. 69, No. 1, 2008, pp. 3153-3159. doi:10.1016/j.na.2007.09.008

[26] E. A. Ddas, M. Benchohra and S. Hamani, "Impulsive Fractional Differential Inclusions Involving the Caputo Fractional Derivative,” Fractional Calculus \& Applied Analysis, Vol. 12, No. 1, 2009, pp. 15-36.

[27] J. Henderson and A. Ouahab, "Impulsive Differential Inclusions with Fractional Order,” Computers \& Mathematics with Applications, Vol. 59, No. 3, 2010, pp. 11911226. doi:10.1016/j.camwa.2009.05.011

[28] A. Pazy, "Semigroups of Linear Operators and Applications to Partial Differential Equations," Springer Verlag, New York, 1983.

[29] J. P. Aubin and H. Frankoeska, "Set-Valued Analysis," Birkhäuser, Boston, Basel, Berlin, 1990.

[30] C. Castaing and M. Valadier, "Convex Analysis and Measurable Multifunctions," Lecture Notes in Mathematics, Springer Verlag, Berlin and New York, 1977.

[31] S. Hu and N. S. Papageorgiou, "Handbook of Multival- ued Analysis. Vol. I: Theory in Mathematics and Its Applications, Vol. 419,” Kluwer Academic Publisher, Dordrecht, 1979.

[32] S. Hu and N. S. Papageorgiou, "Handbook of Multivalued Analysis. Vol. II: Theory in Mathematics and Its ApPlications, Vol. 500, Kluwer Academic Publisher, Dordrecht, 2000.

[33] M. Kamenskii, V. Obukhowskii and P. Zecca, "Condensing Multivalued Maps and Semilinear Differential Inclusions in Banach Spaces,” De Gruyter Series in Nonlinear Analysis and Applications 7, Walter, Berlin, 2001.

[34] H. F. Bohnenblust and S. Karlin, "On a Theorem of Ville, in: Contribution to the Theory of Games," Princeton University Press, Princeton, 1950, pp. 155-160.

[35] H. Covitz and S. B. Nadler, "Multivalued Contraction Mapping in Generalized Metric Space,” Israel Journal of Mathematics, Vol. 8, No. 1, 1970, pp. 5-11.

[36] A. Granass and J. Dugundij, "Fixed Point Theorems," Springer-Verlag, New York, 2003.

[37] F. Hiai and H. Umegaki, "Integrals, Conditional Expectation, and Martingales of Multivalued Functions,” Journal of Multivariate Analysis, Vol. 7, No. 1, 1977, pp. 149182.

[38] A. Bressan and G. Coombo, "Extensions and Selections of Maps with Decomposable Values," Studia Mathematica, Vol. 90, 1, 1988, pp. 69-86. 